\title{
Construction and Effect Evaluation of Engineering Imaginative Teaching Model
}

Yaonan Lin, Department of Business Administration, Fu Jen Catholic University

Fa-Chung Chiu. Department of Counseling Psychology, Chinese Culture University

Hsueh-Chih Chen, Department of Educational Psychology and Counseling, National Taiwan Normal University

Yu-Lin Chang, Department of Educational Psychology and Counseling, National Taiwan Normal University

Chi-Ling Kao, Department of Leisure Business Management, Hung Kuo Delin University of Technology

Imagination covers divergent and convergent thinking. Divergent thinking implies that one person can propose multiple or unique solutions for a problem or task (Guilford, 1967). An example is Guilford's Alternative Uses Test, in which participants must create as many uses for a simple object (e.g., a pen) as they can think of. Test results are evaluated according to the indicators of fluency (i.e., the ability to generate numerous ideas), originality (i.e., the ability to generate novel ideas), and flexibility (i.e., the ability to generate ideas for several conceptual categories)

Furthermore, imagination is a fundament is a fundamental ability, particularly given the numerous temptations they are faced with in society. To live a successful and happy life, people must possess some ability to resist temptation (Huang, Liu, \& Zhu, 2015); thus, exercising Imagination (Wilson, Sayette, \& Fiez, 2014,) is a crucial ability. Previous studies have explored how individuals' ability to exercise imagination influences their divergent and convergent thinking. Regarding convergent thinking, previous studies have shown that after exercising imagination, people become less able to solve analytical problems (Schmeichel, Vohs, \& Baumeister 2003) and anagrams (i.e.,convergent

thinking; Baumeister, Bratslavsky, Muraven, \& Tice, 1998). However, Chiu (2014) found that after exercising imagination, people exhibit enhanced ability to improve divergent thinking, indicating that exercising imagination influences divergent and convergent thinking differently.

The purpose of this study is to develop an engineering imagination teaching model and to design a curriculum and test the effectiveness of the course. In this study, college students were assigned to the engineering imagination group and the control group in the pre- and post- tests. The experimental procedures of this study are as follows: 1 . In the pretest, both conditions received the technological imagination disposition scale and the Imaginative Thinking Tests; 2 . The participants in the imaginative group attended a 30-hour imaginative curriculum, and the participants in the control group attended a 30-hour psychology-related curriculum.

Table 1 Means and standard deviations of technological imagination disposition for MEI and control groups

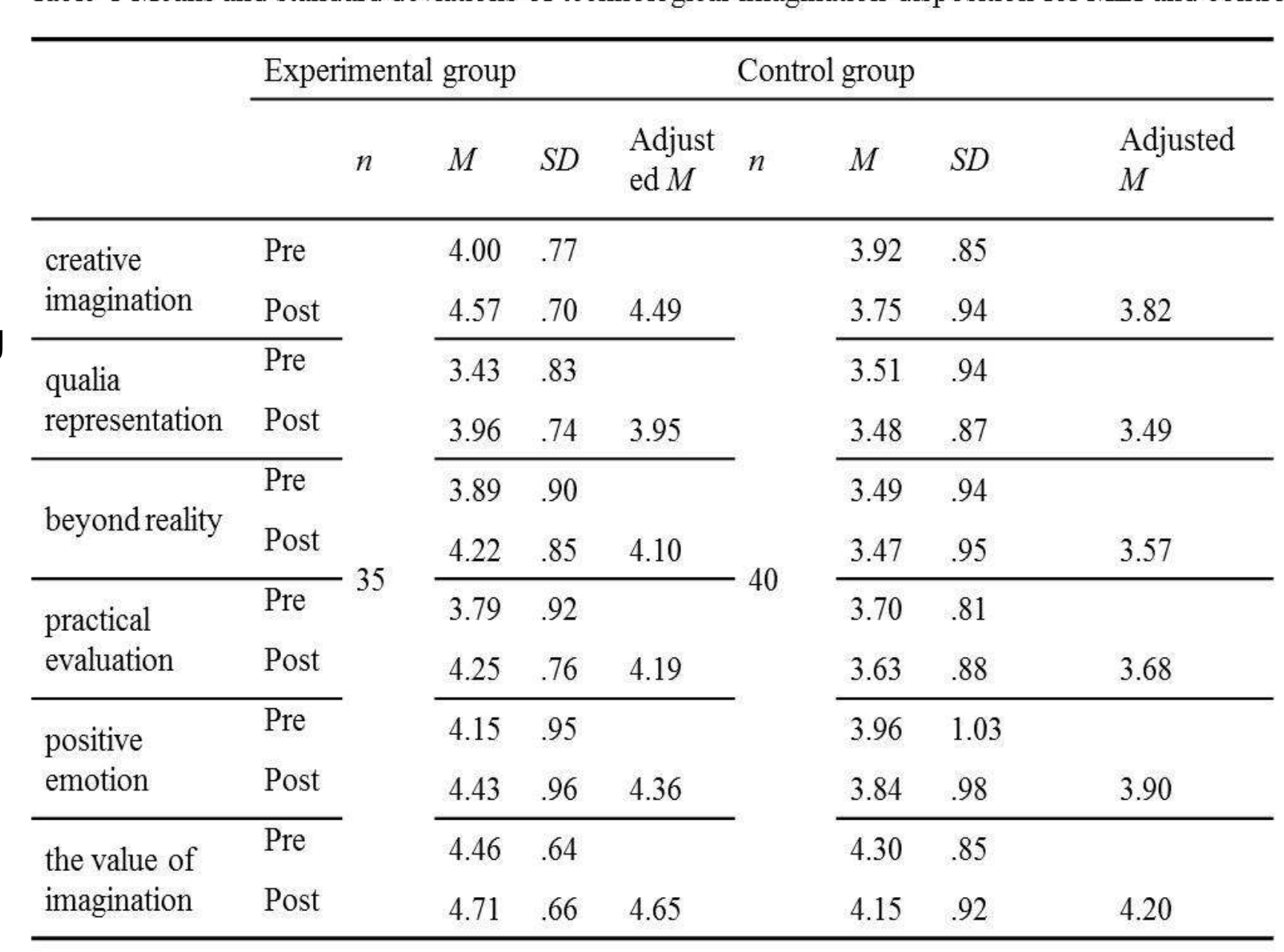

In the post-test, both groups received the technological imagination disposition scale and the Imaginative Thinking Tests. The results are as follows: 1. Comparing with the control group, the imagination group showed improved effect on creative imagination, qualia representation, beyond reality, practical evaluation, positive emotion, and value indicators of the technological imagination disposition scale; 2. Participants in the imaginative condition showed improvement in fluency, beyond reality, and flexibility indicators; however, there was no significant improvement in the vividness indicator.
Additionally, qualitative analysis revealed that the participants in the imaginative group obtained the concept of creative thinking their thinking mode and the attitude of life changed; they acquired imaginative thinking skills. In summary, the engineering imagination curriculum developed in this study is effective and can be applied to the practice of engineering imaginative thinking curriculum.
Table 2 The post-hoc comparison of the means of technological imagination disposition for MEI and control groups

\begin{tabular}{|c|c|c|c|c|c|}
\hline \multirow{2}{*}{ Subscale } & \multirow{2}{*}{$\begin{array}{l}\text { Mean } \\
\text { difference }\end{array}$} & \multirow[b]{2}{*}{$t$} & \multirow[b]{2}{*}{$p$} & \multicolumn{2}{|c|}{$\begin{array}{l}95 \% \text { confidence } \\
\text { intervals }\end{array}$} \\
\hline & & & & $\begin{array}{l}\text { Lower } \\
\text { bound }\end{array}$ & $\begin{array}{l}\text { Upper } \\
\text { bound }\end{array}$ \\
\hline creative imagination & .67 & 4.39 & .001 & .36 & .97 \\
\hline qualia representation & .46 & 3.00 & .003 & .16 & .78 \\
\hline beyond reality & .53 & 2.94 & .005 & .17 & .89 \\
\hline practical evaluation & .51 & 3.23 & .002 & .19 & .82 \\
\hline positive emotion & .46 & 2.59 & 012 & .11 & .82 \\
\hline $\begin{array}{l}\text { the value of } \\
\text { imagination }\end{array}$ & .45 & 3.00 & .004 & .15 & .75 \\
\hline
\end{tabular}

- 